\section{The superficial venous pump}

\author{
Konstantin Mazayshvili \\ Surgical Department, Surgut State \\ University, Russia
}

\begin{abstract}
The present study has revealed the relationship between the cross sectional area of the great saphenous vein and the degree of tension in the superficial fascia of the thigh. We conducted an ultrasound examination with 27 patients (54 lower limbs) in both standing and walking positions. With an increase and decrease in the degree of tension of the superficial fascia, the blood is pushed to the sapheno-femoral junction. Nearly $200 \mathrm{~mm}^{3}$ of blood flows in, and is pushed out of, a 100-mm great saphenous vein segment in the thigh, towards the sapheno-femoral junction during a step cycle. As a result, the active function of the fascial compartment of the great saphenous vein has been found. We have called this mechanism the superficial venous pump.
\end{abstract}

\section{Introduction}

The main functions of the venous system are: to return blood to the heart, and to serve as a capacitance in order to maintain the filling of the heart. Motive forces in the venous return are common knowledge in medical literature, but are limited to respiration and skeletal muscle contractions of the extremities.

Miller et al. ${ }^{1}$ determined that phasic blood flow coincides with breath. The respiratory modulation of venous return from the lower limb is dependent not only on the pressures produced by the respiratory and skeletal muscle pumps, but is also critically dependent upon the capacitance and compliance of the venous vasculature which separates them. The compliance is the ratio of the change in volume resulting from a change in transmural distending pressure. Because venous compliance is very high at low pressures, this is one of the main factors of the pumping mechanism itself. The peripheral venous pump is able to perform its main function of moving blood to the heart by the contraction of muscles in the foot, and the compression of the plantar vein when pressure is applied to the foot. ${ }^{2}$ Ricci et al. ${ }^{3}$ considered the compression of the deep plantar arch, located between the bone arch of the foot and the plantar aponeurosis, as foot systole. Elsner et al. ${ }^{4}$ have contributed data to the research of the venous pump function, namely that the medial outflow tract from the deep plantar venous plexus is fixed by fibrous bands connected to the first metatarsophalangeal joint. In addition, they were able to deduce that passive movements in the joint lead up to a 55 percent increase in blood flow; active movements result in an increase of up to 78 percent.

In 1995, Staubesand et al. investigated the so-called ankle pump. With the aid of duplex ultrasound they measured the rate of blood flow in the GSV of the lower extremity during a relaxed state, as well as during active and passive movements of the ankle joint. As a result of their observations, they reached the conclusion that movements in the ankle joint significantly increase the rate of blood flow in the GSV. ${ }^{5}$ During movement the muscles contract, resulting in blood being pushed away from the leg veins to the popliteal vein, which rapidly increases blood flow. As a result of the arising pressure gradient, distal valves in the deep and communicant veins close, thus preventing backflow. When the muscles in the calf are relaxed, the pressure created within the deeper veins is less than that in the superficial venous system, and blood repeatedly accumulates and leaves there through perforating veins and muscular branches. ${ }^{6}$ The muscles of the venous pump also participate in preventing orthostatic intolerance by enhancing the venous return. ${ }^{7}$ The muscular venous pump in each of the lower extremity segments ensures significant acceleration of blood flow in the deep veins of the lower extremities. Meanwhile, little data is available concerning the relation between the pumping mechanism and the variation of superficial venous outflow in the superficial GSV.

The course of the GSV in a fascial canal derived from the deep fascia of the lower extremity is described..$^{8,9}$ The fascial canal is a sheath surrounding the GSV, and is continuous from the thigh to the leg (Figure 1). As described, it supports the vein from the hydrostatic pressure of the blood and prevents its dilation. ${ }^{8}$ The malfunction of this fascial envelope of the vein, in whole or in part, may be involved in the pathogenesis or complications in the varicosities, of the GSV.

Ultrasonic examination of the GSV topography demonstrates that the GSV lies between sheets of saphenous fascia that are loosely adherent to its wall (Figure 2).

Figure 2 shows that there is tissue space between the GSV and the sheets of saphenous fascia, and that the fascia rests on a small segment of the venous wall. This study aims at identifying mechanisms of
Correspondence: Konstantin Mazayshvili, Surgical Department, Surgut State University, Russia. E-mail: nmsp1322@gmail.com

Key words: Superficial venous pump; great saphenous vein; venous outflow.

Received for publication: 31 July 2017.

Revision received: 28 November 2017.

Accepted for publication: 28 November 2017.

This work is licensed under a Creative Commons Attribution 4.0 License (by-nc 4.0).

CCopyright K. Mazayshvili, 2018

Licensee PAGEPress, Italy

Veins and Lymphatics 2018; 7:7280

doi:10.4081/vl.2018.7280

effect of the saphenous fascia on the GSV diameter and, therefore, on expelling blood from the limb. This help understanding of how the venous vessel network can optimize blood flow in response to various mechanical forces.

\section{Materials and Methods}

Our investigation was approved by the local ethics committee the National Medical and Surgical Center, Russia. This study was performed in order to determine the role of the saphenous fascial compartment in the venous outflow of the GSV. We used duplex ultrasound to analyze types of GSV location in the thigh (I-type: a single GSV in the saphenous compartment without branching; h-type: GSV in the saphenous compartment with a tributary branching out of the compartment; S-type: GSV is not visible, but there is a tributary vein which is not in the saphenous compartment ${ }^{10}$ and the effect of saphenous fascia on the GSV diameter. For this study, linear transducers with a frequency range of 7.5-13.0 MHz, were used.

A total of 54 limbs in 27 consecutive patients ( 3 male and 24 female) were observed. All patients were Caucasians, with a mean age of 43.0 (SD 14.8) years. The CEAP clinical class for these patients was: $\mathrm{C} 0$ in 3 limbs, $\mathrm{C} 1$ in 28 limbs, $\mathrm{C} 2$ in 16 limbs, C3 in 3 limbs and C4 in 4 limbs. The data was analyzed with StatSoft's Statistica 6.0 , by using a dependent t-test for physical properties. A P value of less than 0.05 was considered statistically significant for all tests.

A reflux in the thigh segment was found in 15 limbs; no reflux was found in 39 limbs. Reflux defined as retrograde flow lasting for more than $0.5 \mathrm{~s}$, whereas less than $0.5 \mathrm{~s}$ is defined as normal or no 
reflux11. Patients who participated in the study were asked to imitate walking movements during their examinations. In the first stage, the GSV diameter was measured in the standing position, with the knee fully extended; this produced the highest degree of tension in the saphenous fascia (Figure $3)$.

The second stage of the patient examination was carried out in the standing position, with the knee flexed (imitating walking movement); this produced a relaxation of the fascial compartment (Figure 4). In order to replicate a working process, we asked the patients to lift one knee up until calf and thigh forms a right angle. After that, patients lowered that leg and repeated with the other leg.

The effect of the saphenous fascia on the GSV diameter was analyzed during two different phases of the step cycle. The GSV diameter was measured at four different levels: at the sapheno-femoral junction (Level 1 ); in the lower third of the thigh (Level 2); in the upper third of the calf (Level 3); in the lower third of the calf (Level 4). The GSV diameter was measured in the vertical position, transversal to vein axis.

The vein capacity was calculated with a theoretically selected GSV segment of 100 $\mathrm{mm}$ in the middle third of the thigh. To simplify the model, it was assumed that in such a segment of the GSV, differences in blood vessel configurations could be ignored. The selected vein segment for our purposes was considered to be cylindrical. The cross-sectional area of the GSV, and the volume of blood it contained, were calculated at two phases of the step cycle: i) during maximum

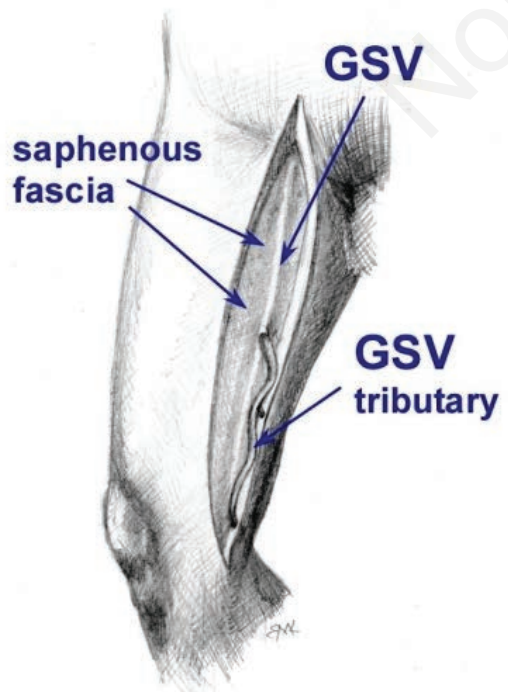

Figure 1. Anatomic relationship between the GSV and the fascial compartment. tension of the saphenous fascia and, consequently, maximum compression of the GSV; ii) during maximum relaxation of the fascial compartment, when the GSV cross section assumed the form of a circle. The tension of the fascia was not measured itself. The assessment point of the SFJ was standardized according to Coleridge-Smith et al. ${ }^{12}$ The cross-sectional area of the blood vessel was calculated utilizing the basic formula to determine the area of an ellipse:

$$
S=\left(\frac{a}{2} \cdot \frac{b}{2}\right) \pi
$$

where $S$ is the GSV cross sectional area; $a$ is the minimum GSV diameter; $b$ is the maximum GSV diameter.

The blood volume within the selected GSV segment was calculated utilizing the formula for the volume of a cylinder:

$$
V=S \cdot H
$$

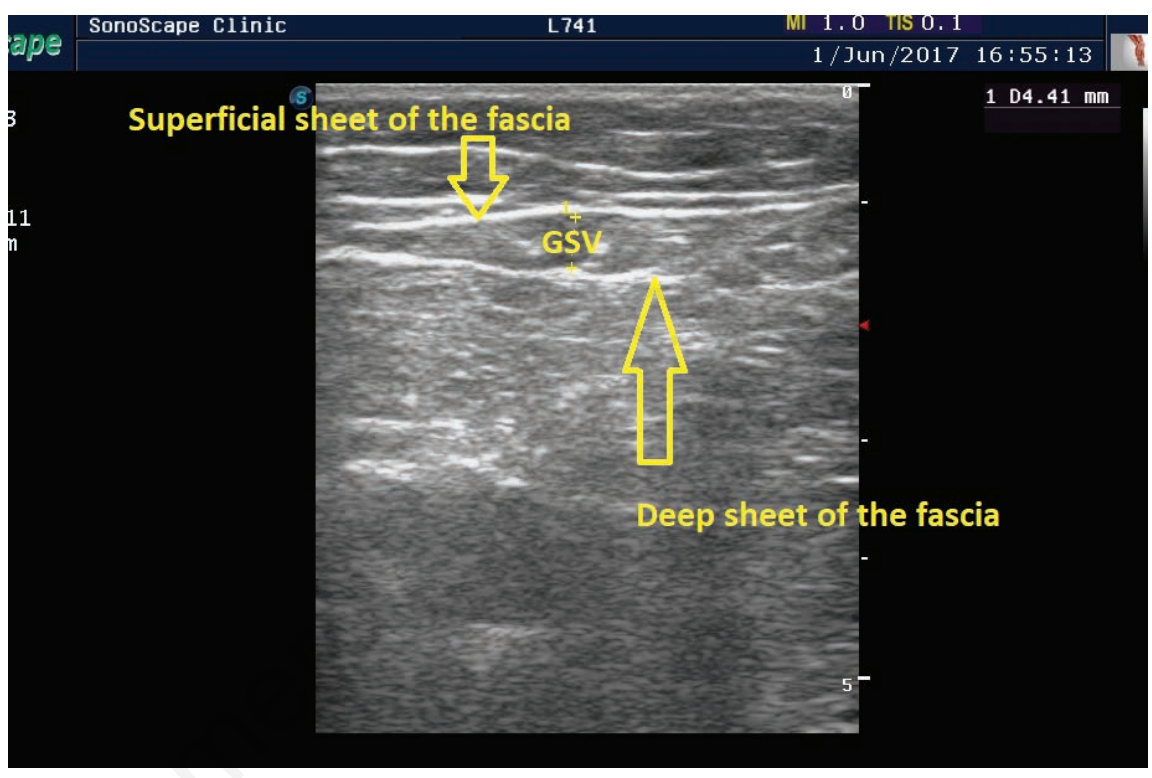

Figure 2. Ultrasonic image of the GSV and its fascial compartment.

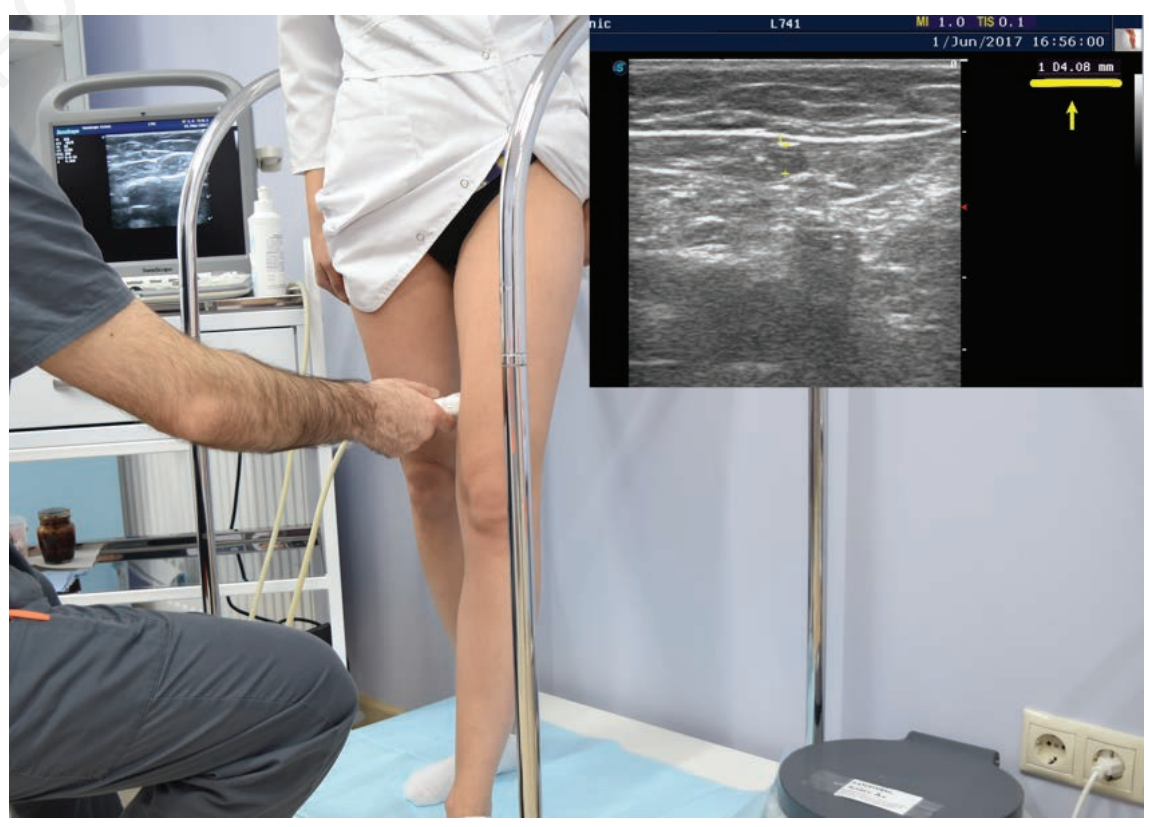

Figure 3. Patient examination in the midstance position of the step cycle. 
where $V$ is the volume of the selected GSV segment; $H$ is the length of the selected GSV segment; $S$ is the GSV cross sectional area.

Based on the previous two results, we calculated the blood volume forced out of the selected GSV segment during the step cycle, due to compression of the GSV by the walls of the fascial compartment. The calculations were based on the following formula:

$\Delta V=V_{1}-V_{2}$

where $\Delta V$ is the blood volume pushed out during the step cycle; $\mathrm{V}_{1}$ is the blood volume in the GSV segment during maximum relaxation of the fascial compartment; $V_{2}$ is the blood volume in the GSV segment during maximum tension of the fascial compartment.

\section{Results and Discussion}

The results of this study show that maximum fascial tension is reached during the midstance position of the step cycle, which results in GSV compression. This occurs because when tense, the sheets of saphenous fascia shift toward each other and cause compression of the GSV (Figure 5).

As compression of the fascia compartment takes place, blood is simultaneously pushed out of the GSV segment towards the sapheno-femoral junction (Level 1). During the heel off position of the step cycle, fascial tension dissipates, and the saphenous fascia tissue no longer exerts pressure on the GSV (Figure 6); this results in the GSV assuming a form resembling a circle. The relaxing fascial compartment allows blood inflow from lower leg segments.

Each process is repeated during subsequent cycles of the step cycle. Changes in the GSV lumen size, in relation to the midstance and heel off positions of the step cycle, are shown in Figure 7.

Formula 2, as previously discussed, was used to calculate the blood volume contained in a $100 \mathrm{~mm}$ segment of a theoretically selected GSV, located in the middle of the thigh. Figure 8 shows these blood volumes in the midstance and step off positions of the step cycle. The calculation does not include the resting superficial vein flow and its velocity.

Using this data in Formula 3, as previously discussed, we obtain the following result: $198.8 \pm 31.2 \mathrm{~mm}^{3}$ of blood flows in, and is pushed out of, a $100 \mathrm{~mm}$ GSV segment in the thigh, towards the saphenofemoral junction (Level 1) during the step cycle. As a result of this research, we have obtained data proving an active function of the GSV fascial compartment, which is the main part of the active mechanism. One of its purposes is to form, together with the GSV trunk, a venous pump, or the superfi- cial venous pump of the lower extremities. The superficial venous pump was recently described. Franceschi and Zamboni distinguished this mechanism and its place in venous outflow from the leg. ${ }^{13}$

Gianesini et al. ${ }^{14}$ determined the flow

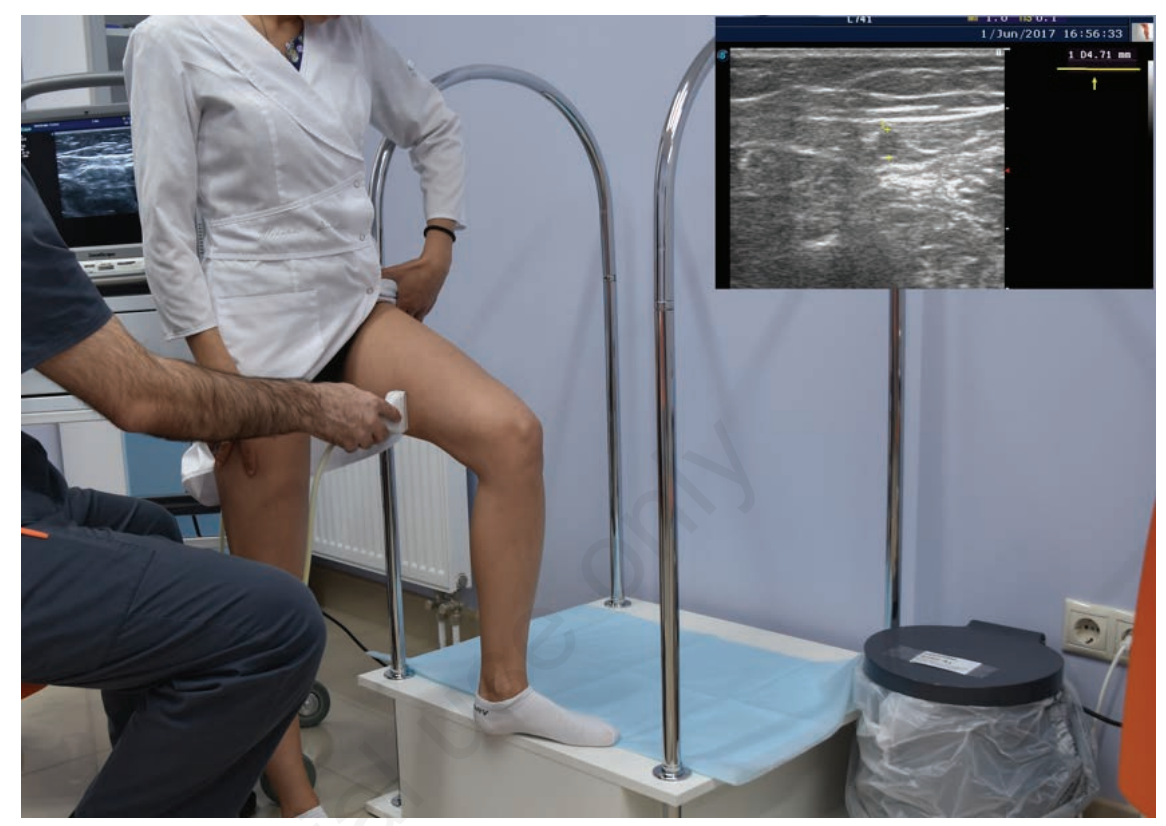

Figure 4. Patient examination in the heel off position of the step cycle.

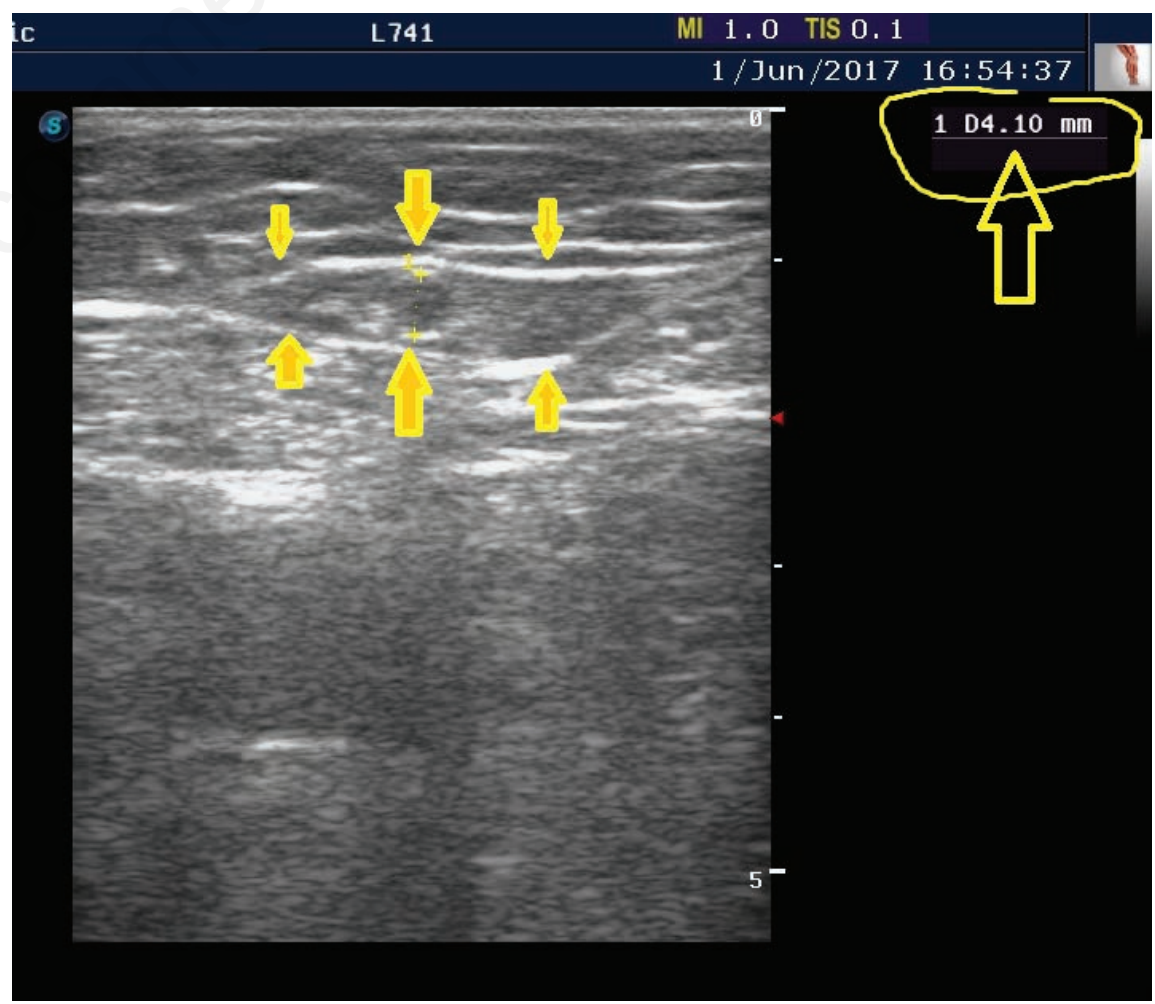

Figure 5. The sheets of saphenous fascia shift toward each other and cause compression of the GSV. 
velocities along the venous segments of the lower limb in 26 healthy volunteers. The peak systolic velocity, average time velocity and diameter of the saphenous system were obtained. The investigation provides evidences of the superficial venous pump as the active mechanism of outflow with the Venturi effect as a potential factor in the flow aspiration from the superficial to the deeper veins.

There was no difference in the results between the group patients with GSV reflux

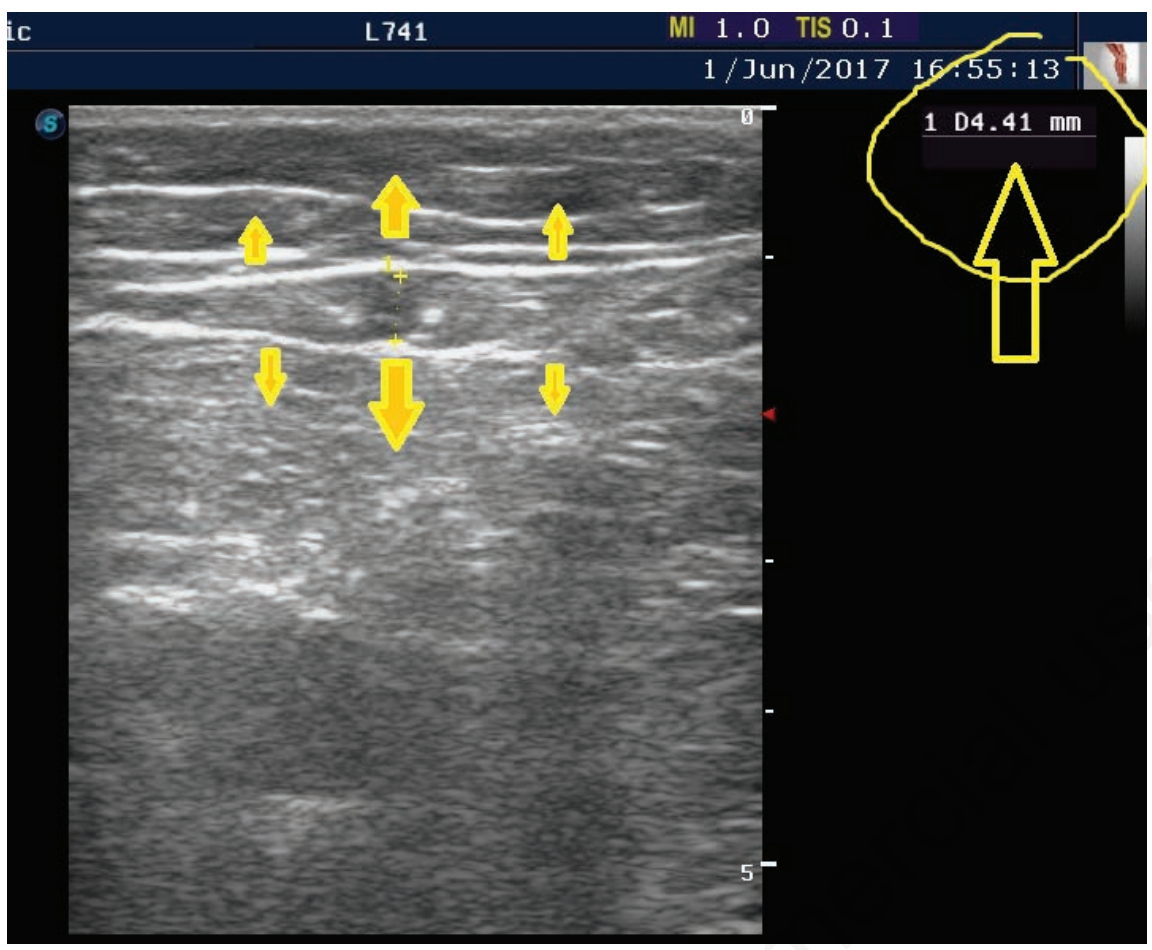

Figure 6. The saphenous fascia tissue no longer exerts pressure on the GSV this results in the GSV assuming a form resembling a circle. and does without. Different risks of its varicose transformation can be assumed depending on the types of GSV location in the thigh (I-type, h-type, S-type). In the htype and S-type of GSV location, in which the trunk lies extrafascially, the sheets of saphenous fascia do not exert pressure on the vein. Meanwhile results of our investigation did not represent differences between the patients with different GSV location in the thigh.

The pumping mechanism can lower the venous pressures and reduce the volume of blood contained within the superficial veins. The tributaries play the role of reservoirs, from which blood enters the GSV. Because of the presence of valves, blood does not return to them during the tension of the saphenous fascia. However, when the valves in the tributaries are incompetent, at the time of compression of GSV in the fascial compartment the blood may overfill them. This can lead to their permanent overstretch and varicose transformation. The $h$ and $s$ anatomical types of GSV location in the thigh also could impair this powerful pump and thus worsen venous return, causing the development of varicose transformation.

The main limitations of the present study are represented by the exclusive focus on the mechanic aspects of venous return. Although this is the main hemodynamic component, the study does not take into account the effects of the vasoactive agents on the saphenous wall as well as the role of the inflammatory cascade on the veins and the surrounding tissues, including the superficial fascia. ${ }^{15-18}$

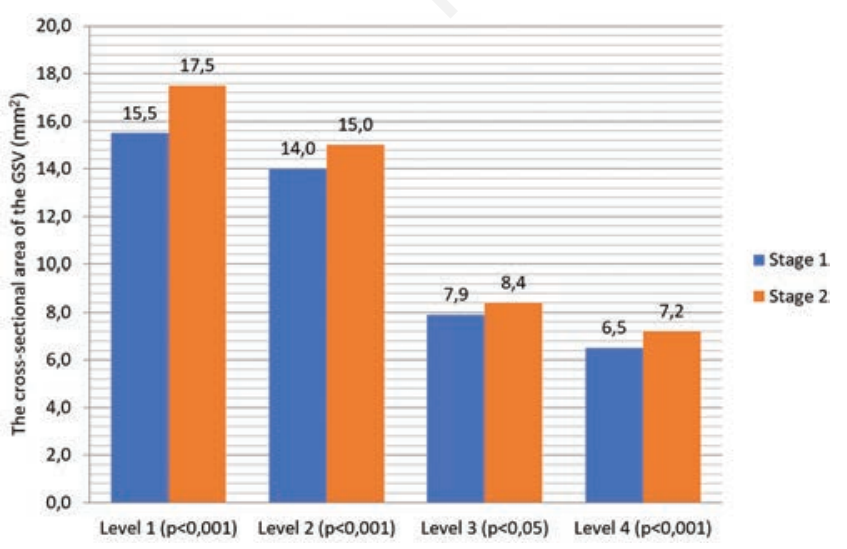

Figure 7. Changes in GSV lumen size in relation to measured positions of the step cycle. Positions of the step cycle: Stage $1=$ midstance; Stage 2 = step off.

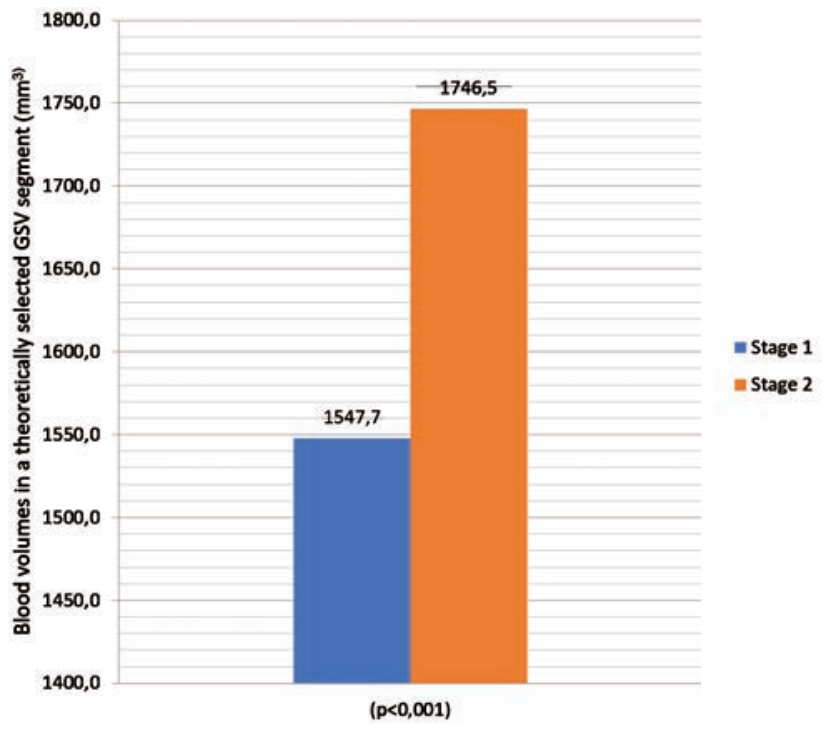

Figure 8. Blood volumes in a theoretically selected GSV segment. Stage 1 = midstance; Stage 2 = step off. 


\section{Conclusions}

The conducted research has revealed an active function of the GSV fascial compartment in causing blood outflow. With the alternating tension and relaxation of the fascial compartment surrounding the GSV, blood is pushed out of the vein segment towards the sapheno-femoral junction, and then filled again, by a two-step process: i) when the saphenous fascia becomes tense, venous walls shift towards each other in the long segment, which leads to a rapid increase in blood flow; ii) when the sheets of saphenous fascia are relaxed, the vein, due to its elasticity, becomes round in its cross section. A concurrent increase in vein volume causes suction of blood to this segment. One-way centripetal blood flow is provided by valves. We have called this mechanism the superficial venous pump. It plays the role of a peripheral superficial heart, which combined with venous valves serve to avoid gravitational reflux during fascial diastole. Finally this is a further argument in favor of saphenous vein sparing strategies. ${ }^{13,16,19}$

We are aware that the study was somewhat biased. A wider range of patients might have provided a more precise understanding of our research. Nevertheless, we hope that it will help in the further study of venous outflow from superficial structures.

\section{References}

1. Miller JD, Pegelow DF, Jacques JA, Dempsey JA. Skeletal muscle pump versus respiratory muscle pump: modulation of venous return from the loco- motor limb in humans. J Physiol 2005; 563:925-43.

2. Uhl JF, Gillot C. Anatomy of the foot venous pump: physiology and influence on chronic venous disease. Phlebology 2012;5:219-30.

3. Ricci S, Moro L, Incalzi AR. The foot venous system: anatomy, physiology and relevance to clinical practice. Dermatol Surg 2014;40:225-33.

4. Elsner A, Schiffer G, Jubel A, et al. The venous pump of the first metatarsophalangeal joint: clinical implications. Foot Ankle Int 2007;8:902-9.

5. Staubesand J, Heisterkamp T, Stege H. Use of Duplex sonography to investigate the effect of active and passive movement at the ankle joint for promoting venous return. Clin Anat 1995;2:96101.

6. Alimi YS, Barthelemy P, Juhan C. Venous pump of the calf: a study of venous and muscular pressures. J Vasc Surg 1994;20:728-35.

7. Stewart JM, Medow MS, Montgomery LD, McLeod K. Decreased skeletal muscle pump activity in patients with postural tachycardia syndrome and low peripheral blood flow. Am J Physiol Heart Circ Physiol 2004;286:H1216-22.

8. Papadopoulos NJ, Sherif MF, Alberte EN. A fascial canal for the great saphenous vein: gross and microanatomical observations. J Anat 1981;132:321-9.

9. Cavezzi A, Labropoulos N, Partsch H, et al. Duplex Ultrasound Investigation of the Veins in Chronic Venous Disease of the Lower Limbs - UIP Consensus Document. Part II. Anatomy. Eur J Vasc Endovasc Surg 2006;31:288-99.

10. Ricci S, Caggiati A. Echoanatomical patterns of the long saphenous vein in patients with primary varices and in healthy subjects. Phlebology 1999;14: 54-8.

11. Labropoulos N, Tassiopoulos AK, Kang SS. Prevalence of deep venous reflux in patients with primary superficial vein incompetence J Vasc Surg 2000;32:6638.

12. Coleridge-Smith P, Labropoulos N, Partsch H, et al. Duplex Ultrasound Investigation of the Veins in Chronic Venous Disease of the Lower Limbs UIP Consensus Document. Part I. Basic Principles Eur J Vasc Endovasc Surg 2006;31:83-92.

13. Franceschi C, Zamboni P. Principles of venous hemodynamics. New York: Nova Science Publishers; 2009.

14. Gianesini S, Sisini F, Di Domenico G, et al. Lower limbs venous kinetics and consequent impact on drainage direction. Phlebology 2018;33:107-14.

15. Rizzi A, Quaglio D, Vasquez G, et al. Effects of vasoactive agents in healthy and diseased human saphenous veins. J Vasc Surg 1998;28:855-61.

16. Zamboni $\mathrm{P}$, Spath $\mathrm{P}$, Tisato $\mathrm{V}$, et al. Oscillatory flow suppression improves inflammation in chronic venous disease. J Surg Res 2016;205:238-45.

17. Tisato V, Zamboni P, Menegatti E, et al. Endothelial PDGF-BB produced ex vivo correlates with relevant hemodynamic parameters in patients affected by chronic venous disease. Cytokine 2013;63:92-6.

18. Zamboni P, Lanzara S, Mascoli F, et al. Inflammation in venous disease. Int Angiol 2008;27:361-9.

19. Mendoza E. Primum non nocere. Veins and Lymphatics 2017;6:6646. 